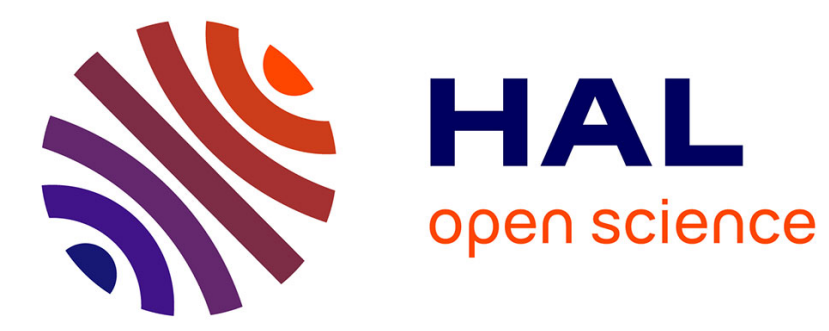

\title{
The influence of pipeline supports stiffness onto the water hammer run
}

\author{
Adam Adamkowski, Slawomir Henclik, Waldemar Janicki, Mariusz \\ Lewandowski
}

\section{- To cite this version:}

Adam Adamkowski, Slawomir Henclik, Waldemar Janicki, Mariusz Lewandowski. The influence of pipeline supports stiffness onto the water hammer run. 16th International Symposium on Transport Phenomena and Dynamics of Rotating Machinery, Apr 2016, Honolulu, United States. hal-01884243

\section{HAL Id: hal-01884243 \\ https://hal.science/hal-01884243}

Submitted on 30 Sep 2018

HAL is a multi-disciplinary open access archive for the deposit and dissemination of scientific research documents, whether they are published or not. The documents may come from teaching and research institutions in France or abroad, or from public or private research centers.
L'archive ouverte pluridisciplinaire HAL, est destinée au dépôt et à la diffusion de documents scientifiques de niveau recherche, publiés ou non, émanant des établissements d'enseignement et de recherche français ou étrangers, des laboratoires publics ou privés. 


\title{
The influence of pipeline supports stiffness onto the water hammer run
}

\author{
Adam Adamkowski ${ }^{1}$, Slawomir Henclik ${ }^{1 \star}$, Waldemar Janicki $^{1}$, Mariusz Lewandowski ${ }^{1}$
}

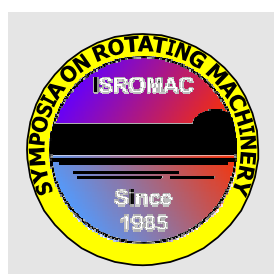

ISROMAC 2016

International

Symposium on Transport

Phenomena and

Dynamics of

Rotating Machinery

Hawaii, Honolulu

April 10-15, 2016

\begin{abstract}
Water hammer $(\mathrm{WH})$ phenomenon may produce various undesired effects in pipelines. Dynamic interaction between the liquid and the structure is known to influence the transient pipe flow parameters in non-rigid systems. One can expect that due to basic energy conservation considerations the energy outflow from the liquid to the elastic structure would result in lowering of WH pressures. However, this effect is not unambiguous and is not clearly and uniquely explained in literature. Thus, its proper understanding is of great practical importance.

In the paper the authors try to examine these effects mainly on the basis of experimental results acquired from measurements at a special test rig designed and constructed at the Institute of FluidFlow Machinery in Gdansk. The main part of the rig is a cooper pipeline of the length of about $59 \mathrm{~m}$, fixed to the floor with a number of elastic supports. WH runs were generated, measured and analyzed for supports of varying stiffness. Pipeline free vibrations induced by mechanical shock were measured as well. The conclusions have been found and they are presented in the paper. Still, a very important part of the job was to find the physical interpretation and explanation of the results, which allows for understanding of that phenomenon.
\end{abstract}

\section{Keywords \\ Pipe transient flow - Water hammer - Fluid-structure interaction - Elastic pipe supports \\ ${ }^{1}$ Department of Hydropower, Institute of Fluid-Flow Machinery PAS, Gdansk, Poland \\ *Corresponding author: shen@imp.gda.pll}

\section{INTRODUCTION}

Water hammer (WH) appears when a (steady) pipe flow conditions are disturbed by any reason, e.g. valves operation or hydraulic machinery load variation. The essence of WH phenomenon is the transfer of liquid kinetic energy to the potential energy of elasticity, which, for weakly compressible liquid may produce high pressure variations. They propagate through the pipe system as elastic waves and may induce perturbations in system functioning. For rigid or quasi-rigid structure (pipeline, supports) these behaviors are well described by the classic WH theory [1] which uses two hyperbolic partial differential equations (PDE) for modeling of the liquid pressure and velocity variations in time and space along one-dimensional pipeline. For elastic structure, which takes part in the energy transfer process the dynamic fluid-structure interaction (FSI) occurs [2]. When the longitudinal pipe motion is going to be taken into account the liquid equations has to be adequately modified. Additional two PDEs are formulated for the pipe motion which finally produces the four equations model of WH-FSI [3]. For a more precise description of the pipe motion additional ten equations are formulated for the transversal and rotational pipe vibrations to form finally the fourteen equations standard model of WH-FSI [3,4]. FSI can be considered to give an opportunity to lower the liquid pressures, just due to general energy conservation consideration. But, as pointed by scientists $[5,6,7]$ this effect is not unambiguous and pressure increase may also happen.
Three main FSI coupling factors are pointed in the literature. The weakest is the friction between the pipewall and the liquid. The second is the Poisson effect that introduces coupling between the liquid pressure variation and pipe longitudinal strains. The third mechanism is the junction coupling (JC) effect that occurs at pipe bends, ends, valves, flow throttling elements and other places where strong FSI can appear. The JC effect is especially important if the pipeline has the ability to move as a whole body, which happens for elastic pipe supports. This pipe motion produces the possibility of energy outflow from the liquid to the structure, especially supports, and dissipation there. Finally this effect can result in additional pressure changes and lowering of pressure magnitudes can be expected. Other causes of energy dissipation like pipewall friction $[8,9]$ or pipe material damping $[10,11]$ will also influence the WH-FSI phenomenon.

In order to examine experimentally the influence of elastic pipe supports onto $\mathrm{WH}$ run a special test rig was designed and constructed at the laboratory hall of the Institute of Fluid-Flow Machinery of the Polish Academy of Sciences (IMP PAN). A series of measurements of WH runs for various pipeline supporting systems were conducted. Other auxiliary measurements were performed as well. The results were preliminary analyzed and compared with theoretical and numerical investigations. The basic conclusions on the effects produced by elastic supporting system of the pipeline have been formulated. Further analyses are still intended. 


\section{EXPERIMENTAL STAND}

The general, functional scheme of the experimental rig is presented in Figure 1. The main part of it is the testing pipeline (1) with transducers and measuring gauges. The pipe flow is driven by the constant pressure of the water-air pressure vessel (3) and the WH is excited by fast closing of the valve (2) at the pipe-end. The rest of the hydraulic part of the set-up with the pump, lower tank, supplying pipes and a system of valves allow for working in stable conditions.

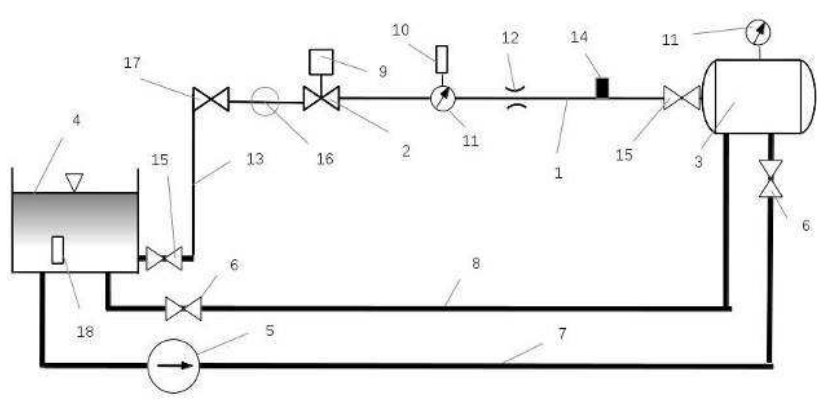

Figure 1. Functional scheme of the test rig

1 - cooper (testing) pipeline, 2 - shut-off ball valve, 3 - water-air pressure vessel, 4 - open tank, 5 - pump, 6 - control valve, 7 - forward pump pipe, 8 - backward pipe, 9 - valve closing drive and closing angle gauge, 10 - temperature gauge, 11 - pressure transducer, 12 ultrasound flow meter, 13 - rubber backward hose, 14 - vibration transducer, 15 - shut-off control valve, 16 - volume meter, 17 - control valve, 18 - temperature meter, 19 - strain gauge (not presented)

The testing pipeline has the length of about $59 \mathrm{~m}$ and it is constructed of several straight cooper pipe reaches joint by knees. Its inner diameter is $D=20 \mathrm{~mm}$ and pipe-wall thickness $e=1 \mathrm{~mm}$. The general scheme of the pipeline is presented in Figure 2 and a picture of it in Figure 3.

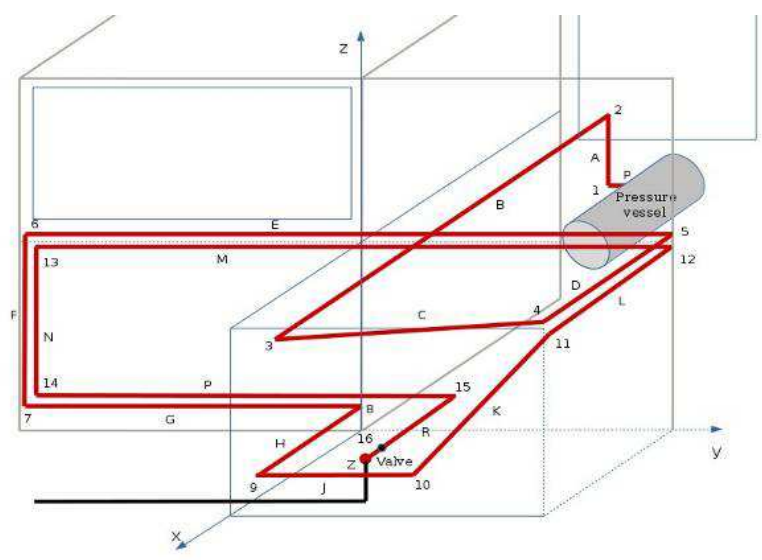

Figure 2. Scheme of the testing pipeline

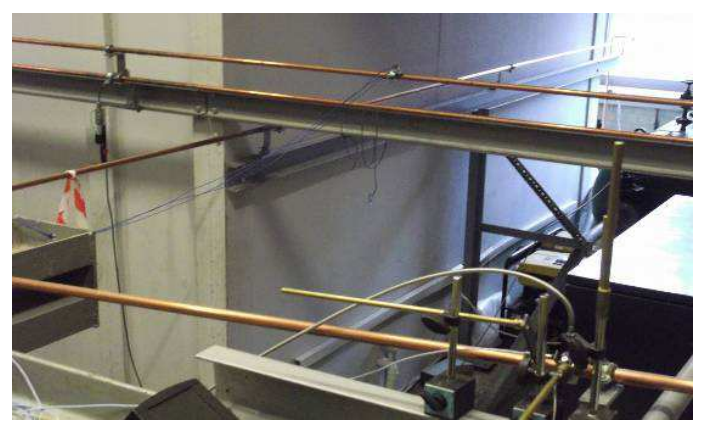

Figure 3. View of the testing pipeline
Article Title -2

In order to induce a repeatable, simple, WH event a special mechanical drive for quick closure of the shut-off valve was constructed. The valve closing time was adjusted at $5 \mathrm{msec}$.

To test the influence of pipe supports stiffness for WH run four types of supports of varying stiffness were designed and constructed at IMP PAN. The supports were made as properly shaped steel flat springs (FS). The views of the supports being used, ordered with increasing stiffness (from left to right) are shown in Figure 4.
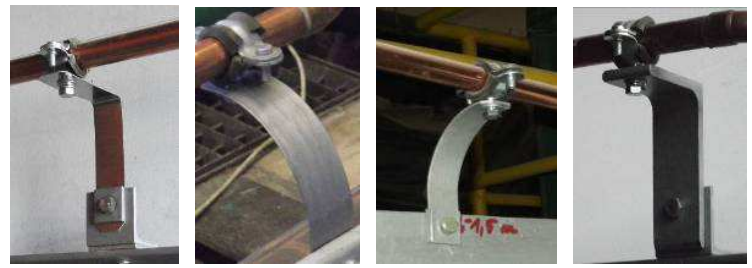

Figure 4. Flat springs (from the left) FS2, FS3, FS4, and FSR for supporting the pipeline

The stiffness matrices of the FS were calculated (selected experimental verification was done as well). For example, the susceptibility coefficients of the supports in vertical direction, were found to be (respectively from FS2 to FSR): $2.1 \mathrm{~mm} / \mathrm{N}$, $0.34 \mathrm{~mm} / \mathrm{N}, 0.085 \mathrm{~mm} / \mathrm{N}, 0.88 \mathrm{~mm} / \mathrm{kN}$. The pipeline was fixed rigidly at both ends and supported with the above supports inside. Two types of support configurations were used - $\mathrm{L}$ (built with 24 elastic supports) and S (25 supports). Within the further discussion we will use a brief denotation, e.g. L3 means configuration $L$ with supports FS3.

Pipe pressures, motions (vibrations), strains and other quantities (flow velocity, temperature) were measured during experiments. Additional tests were conducted as well. Their purpose was to generate free vibrations of the pipeline system with mechanical shock and to observe the transients. A special modal hammer presented in Figure 5 was used for producing a mechanical impact.

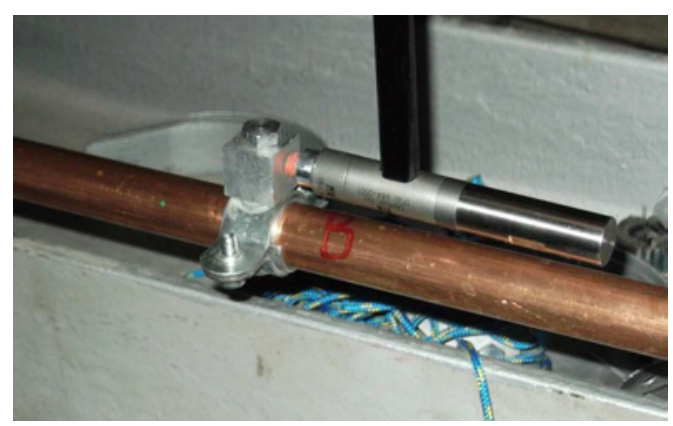

Figure 5. Modal hammer with elastic tip used for generating pipeline transient vibrations

Three types of elastic tips of the hammer were used to produce pulses of various parameters. Hits were generated at precisely determined places and directions. The induced pulses of force were measured.

\section{WATER HAMMER EXPERIMENTS}

The WH runs were conducted for two values of initial pressures of the water-air pressure vessel - higher (denoted as $\mathrm{W}$ ) of $p_{0}=1.12 \mathrm{MPa}$ and lower (denoted $\mathrm{N}$ ) of $p_{0}=0.72$ $\mathrm{MPa}$. For each of them ten values of initial velocities $v_{0}$ 
starting from $0.06 \mathrm{~m} / \mathrm{s}$ up to $2.0 \mathrm{~m} / \mathrm{s}$ were adjusted (they are denoted with numbers $0 . .9$ ). For some of the tests the initial conditions have involved WH with column separation. Eight various support configurations created with four types of supports of different stiffness were applied - L2, L3, L4, LR and S2, S3, S4, SR. Beside of the measurements for fast closing valve additional tests for a handy valve closing were conducted as well. A large number of data were acquired and preliminary analyzed. Further analyses are still intended. In the current paper the analysis is concentrated on the pressure records.

\subsection{Water hammer without column separation}

An example of the results for selected initial conditions of pressure run for the case with no liquid column separation is presented in Figure 6 . The pressures $\mathrm{P} 1$ at the end of the pipeline (close to the valve) for four types of support configurations L2, L3, L4, LR and initial conditions W6 $\left(p_{0}=1.12 \mathrm{MPa}, \mathrm{v}_{0}=0.7 \mathrm{~m} / \mathrm{s}\right)$ are presented.
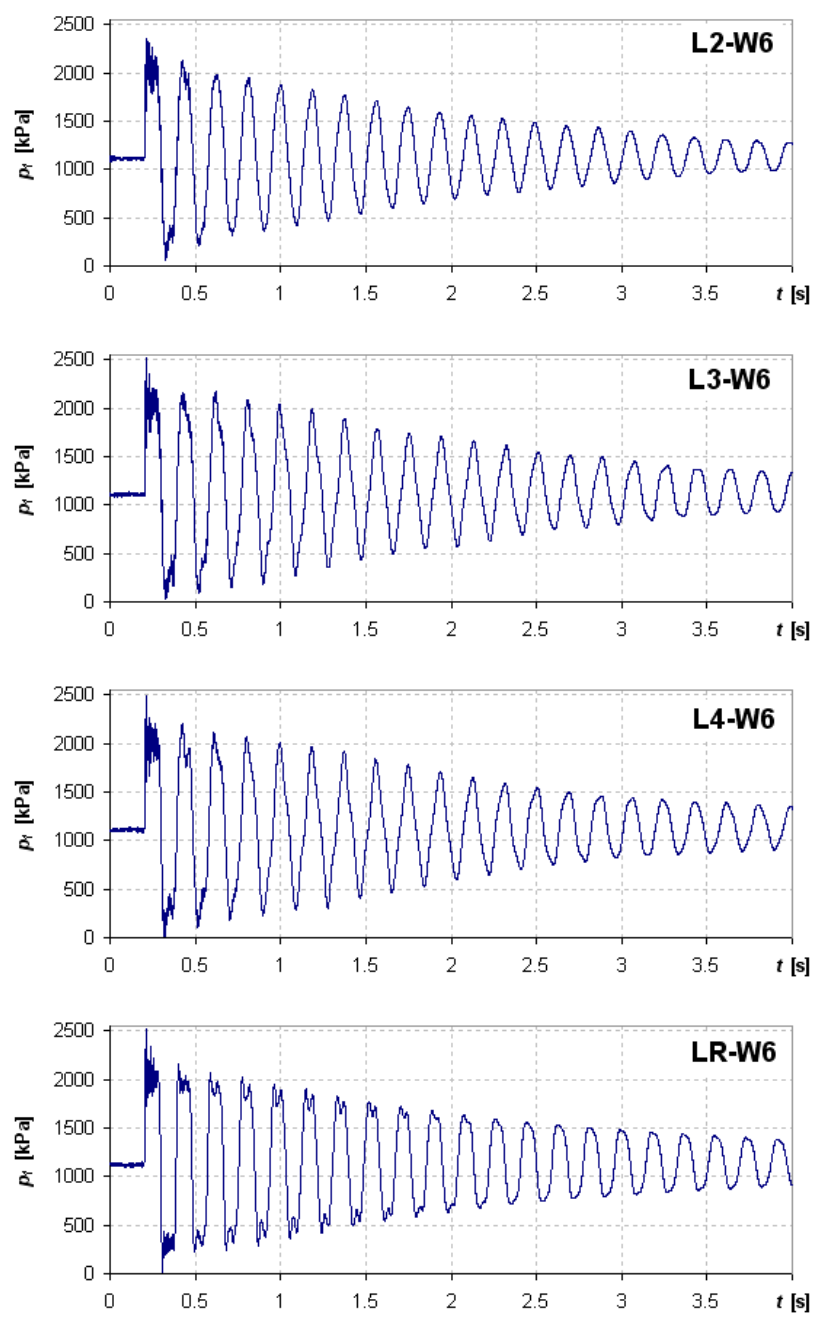

Figure 6. Pressure runs (P1) at the valve for initial flow velocity $0.7 \mathrm{~m} / \mathrm{s}$ and various stiffness of the supports

The high frequency (HF) oscillations observed mainly at the first WH peak are the result of undesired mechanical vibrations produced by the valve closing drive. They are quickly damped for all types of supports - they are present mainly at the first pressure peak and do not influence
Article Title -3

further WH pressure oscillations. It can be also observed that the decaying of pressure oscillations may not be regular at the initial part of the run which is visible mainly at L3 run and slightly at L4. The irregularity is probably the effect of coupled pipeline vibrations and FSI.

The pressures were measured at other points of the pipeline as well. For comparison, pressure runs P3 measured close to the middle of the pipeline $(43 \%$ of the length from the end) for the same initial conditions W6 are presented in Figure 7. The HF disturbances are, in fact, not present there, due to the large distance from the valve. The runs are also more regular in decreasing tendency.
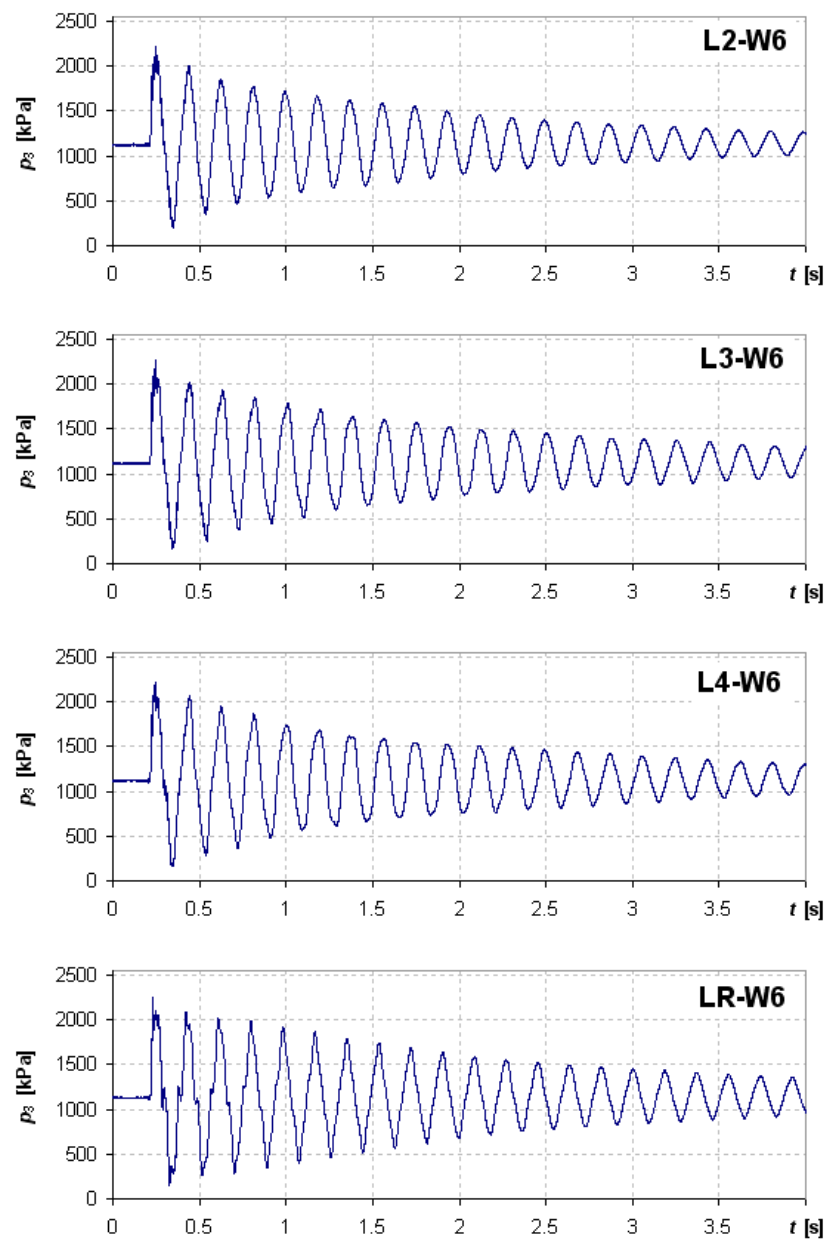

Figure 7. Pressure runs (P3) at the middle part of the pipeline for initial flow velocity $0.7 \mathrm{~m} / \mathrm{s}$ and various stiffness of the supports

\subsection{Preliminary analysis}

Different WH runs can be compared with one another in many ways. In [12] two methods of quantitative analyses basing on pressure wave damping decrement and pressure traces convergence rate were presented. In general a simple method of pressure run assessment can be proposed, which takes into account the pressure changes amplitudes. Two factors should be important - an initial height of the pressure peak and the rate of decaying of the transient. The shape of the waveform should be also important. A logarithmic damping decrement $\delta$ is used herein for estimation of the pressure decaying rate. The average value of $\delta$ for $N$ 
subsequent oscillations, starting from $k$, is calculated with the following formula:

$$
\delta=\frac{1}{N} \ln \left(\frac{p_{k}}{p_{k+N}}\right)
$$

The decrements for the runs presented in Figure 6 were calculated (for $k=5, N>10$ ) and the results were found to be: $\delta_{2}=0.096, \delta_{3}=0.091, \delta_{4}=0.083, \delta_{R}=0.069$. On the basis of the presented results and additional analyses of other runs the following conclusions can be formulated:

- The pressure magnitudes are usually damped more strongly for elastic supports of lower stiffness.

- The initial rectangular shape of the pressure wave is faster transferred to (quasi-) sinusoidal one for supports of lower stiffness.

- The average height of the first pressure peak is nearly the same for all types of supporting systems. However the superimposed HF oscillations generated by the valve drive should be noticed.

- The initial amplitudes may not be damped regularly $(L 3, L 4)$ being for some of the runs higher for latest time moments (as for L3).

- For supports of larger stiffness a slight increase in the celerity of pressure wave is produced. This means the rise in basic $\mathrm{WH}$ frequency of the average value of about $5.4 \mathrm{~Hz}$.

Further analyses, conclusions and an attempt of physical explanation of the results are continued in the section 4.
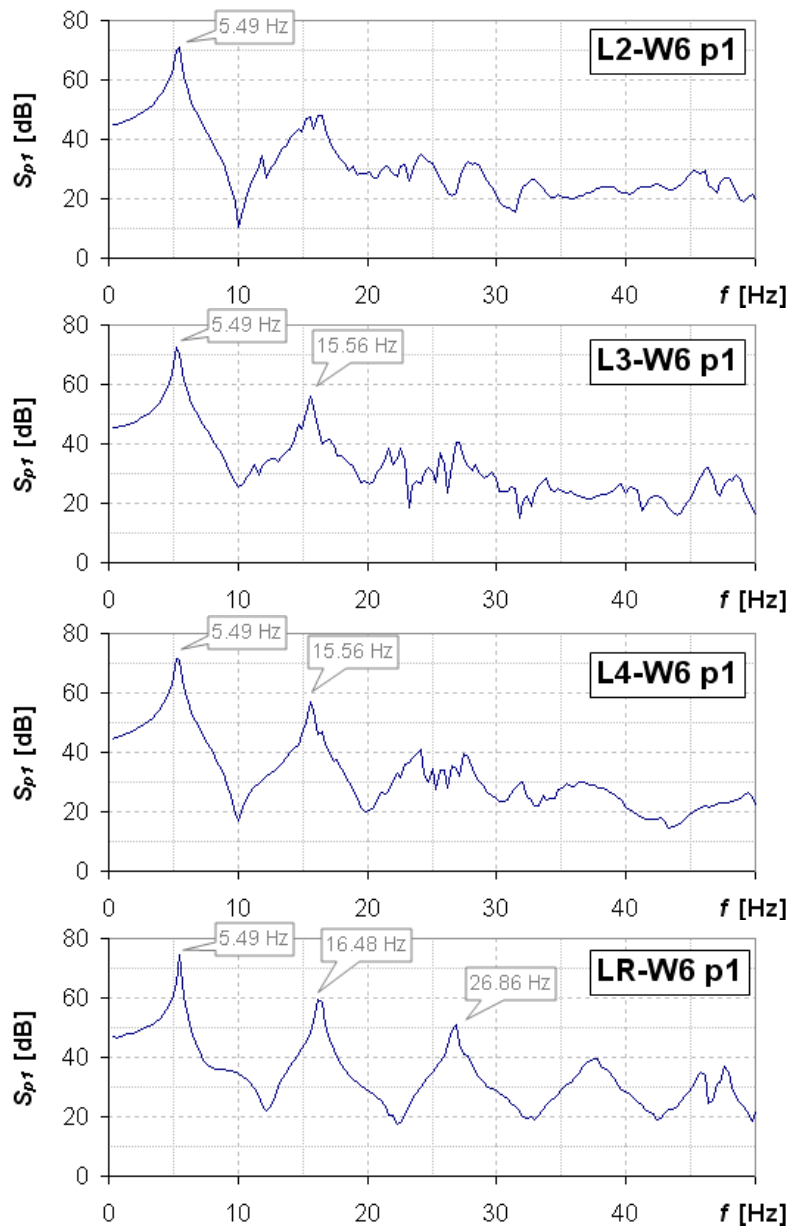

Figure 8. The PDS of pressure signal at the valve for W6 initial condition and various support configuration
Article Title -4

For better understanding of the results additional analysis and processing was done. In Figure 8 the power density spectra (PDS) of the initial $3.2 \mathrm{sec}$. of the runs from Figure 6 are presented. The $\mathrm{dB}$ scale at the diagrams were calculated by taking $10 \log$ of the ratio of the FFT of power signal by the reference level of $0.01 \mathrm{kPa}^{2} / \mathrm{Hz}$. The earlier conclusion of decreasing the pressures magnitudes for lower stiffness of the supports can now be clearly observed. More detailed discussion of these results is included in the section 4.

\subsection{Water hammer with column separation}

For some series of the measurements the initial conditions have produced WH runs with liquid column separation (CS), which appeared when the pressure in the flow dropped close to the saturation value. In Figure 9 the pressures $P 1$ at the valve for four types of support configuration L2, L3, L4, LR and initial conditions $\mathrm{N} 9\left(\mathrm{p}_{0}=0.72 \mathrm{MPa}, \mathrm{v}_{0}=2.0 \mathrm{~m} / \mathrm{s}\right)$ are presented.
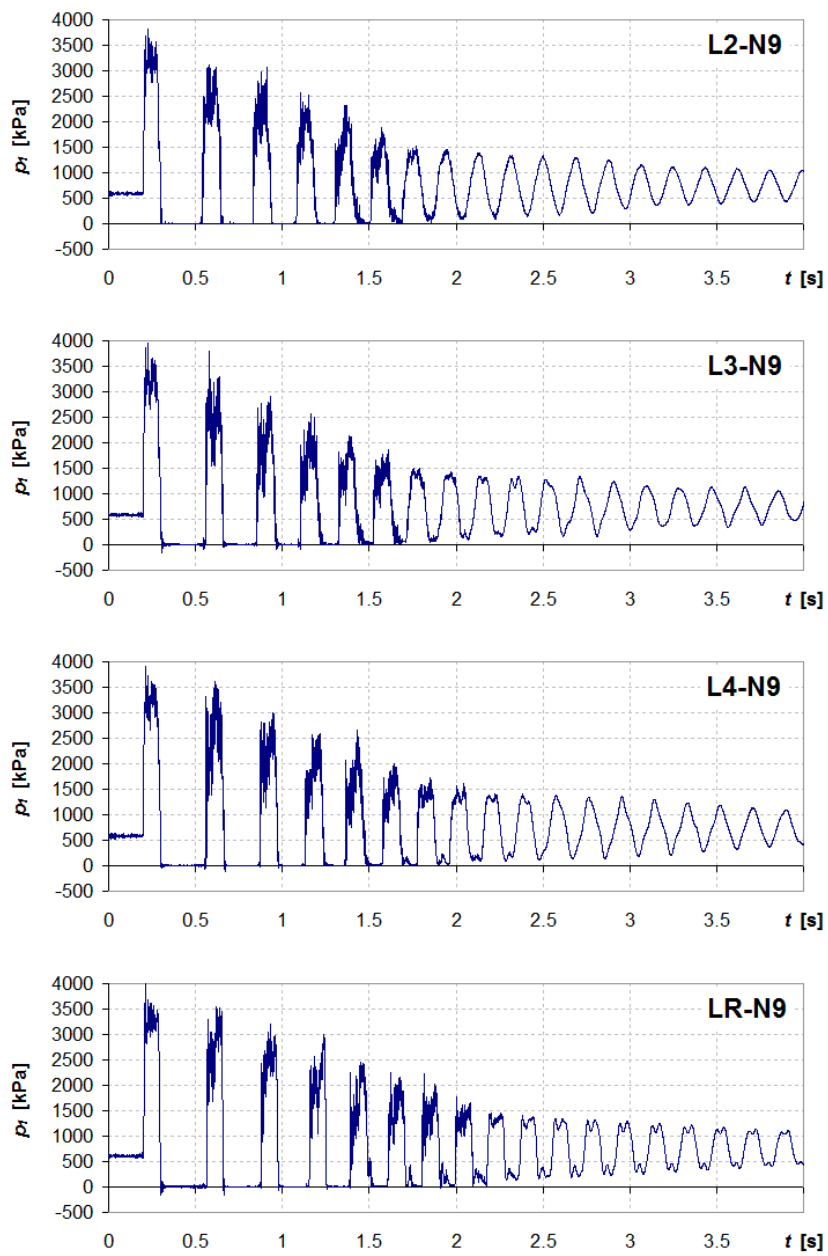

Figure 9. Pressure runs at the valve for initial flow velocity $2.0 \mathrm{~m} / \mathrm{s}$ and various stiffness of the supports

These investigations were not the main purpose of the current research, however preliminary conclusions on the basis of selected results could be formulated. It can be estimated with the Joukovsky formula [1] that column separation effects must appear for the applied initial conditions N9 as the pressure magnitude of $\mathrm{WH}$ wave is about $p_{w H}=2.5 \mathrm{MPa}$. This can be also observed at the diagrams by the analyses of the height of the first pressure 
peak. Thus, as $p_{0}<p_{W H}$, for negative peaks the CS effects appear which can be observed at the diagrams for certain time periods. The HF oscillations at the first pressure peak are, as before, the result of the mechanical excitation from the valve drive. However the subsequent ones are not and they are surely the effect of collapsing cavity bubbles and FSI. Finally the following conclusions are formulated:

- Liquid CS effects are stronger for supports with larger stiffness - the duration of cavity separation is longer.

- The changes in celerity of the pressure wave for various stiffness of the supports are more distinct for WH with CS.

- The HF oscillations superimposed on the main WH pressures are the result of collapsing of cavity bubbles as it was investigated and proved in [13].

\section{PIPELINE TRANSIENT VIBRATIONS}

Additional experiments were conducted as well to get information on characteristics of the system and its other features. The transient vibrations were generated by hitting the pipe with a modal hammer as it is presented in Figure 5. Various hitting methods were applied - three different places $(A, B, C)$ for hitting, five directions $(1,2,3,5,6)$ and three types of the hammer elastic tips ( $k 1, k 2, k 3)$ were used. The $L$ configuration of fixing the pipeline with the four types of elastic supports were tested. Three classes of boundary conditions (BC) were assumed - an empty pipeline (e), a pipeline with flowing water (p) or standing water (s). Specific hydraulic BC were valid for the cases with water. Both valves 15 and 17 (Figure 1) were closed for no-flow case and for flowing water the valve 15 was opened and 17 slightly opened (nearly closed) to produce a very low flow velocity, about $v_{0}=0.2 \mathrm{~m} / \mathrm{s}$. During the transient vibrations all the same quantities as during $\mathrm{WH}$ experiments were recorded (mainly pressures, pipe motions, pipe strains). The liquid pressures and pipe vibrations (accelerations) are discussed herein. The analyses are focused on the power spectrum densities of recorded signals which allowed to find and identify characteristic frequencies of the system.

In Figure 10 the power spectrum density of pressure at the valve for the case of pipeline with standing water (s) and various support stiffness are presented. The initial peaks at the diagrams $(9.77 \mathrm{~Hz}, 18.92 \mathrm{~Hz}$ for $\mathrm{L} 2$ and $10.38 \mathrm{~Hz}, 19.84 \mathrm{~Hz}$ for L3) must be the result of the pressure wave induced by mechanical shock. Those frequencies are consistent (approximately) with the $\mathrm{BC}$ which produce a value twice as large (in fact series of even multiplications) as basic WH frequency. It can be observed the initial peaks are much lower for higher stiffness of the supports which means that $\mathrm{WH}$ effect can be induced by mechanical impact due to junction coupling effect and this phenomenon is much weaker for more rigid pipeline fixing. The change in BC for the pipeline with flowing water (p) gives characteristic frequency similar as for typical $\mathrm{WH}(4.88 \mathrm{~Hz})$ as it is presented in Figure 11. This kind of measurements made it also possible to identify the basic characteristic frequencies of the pipeline system fixed with elastic supports considered as vibrating mechanical structure.
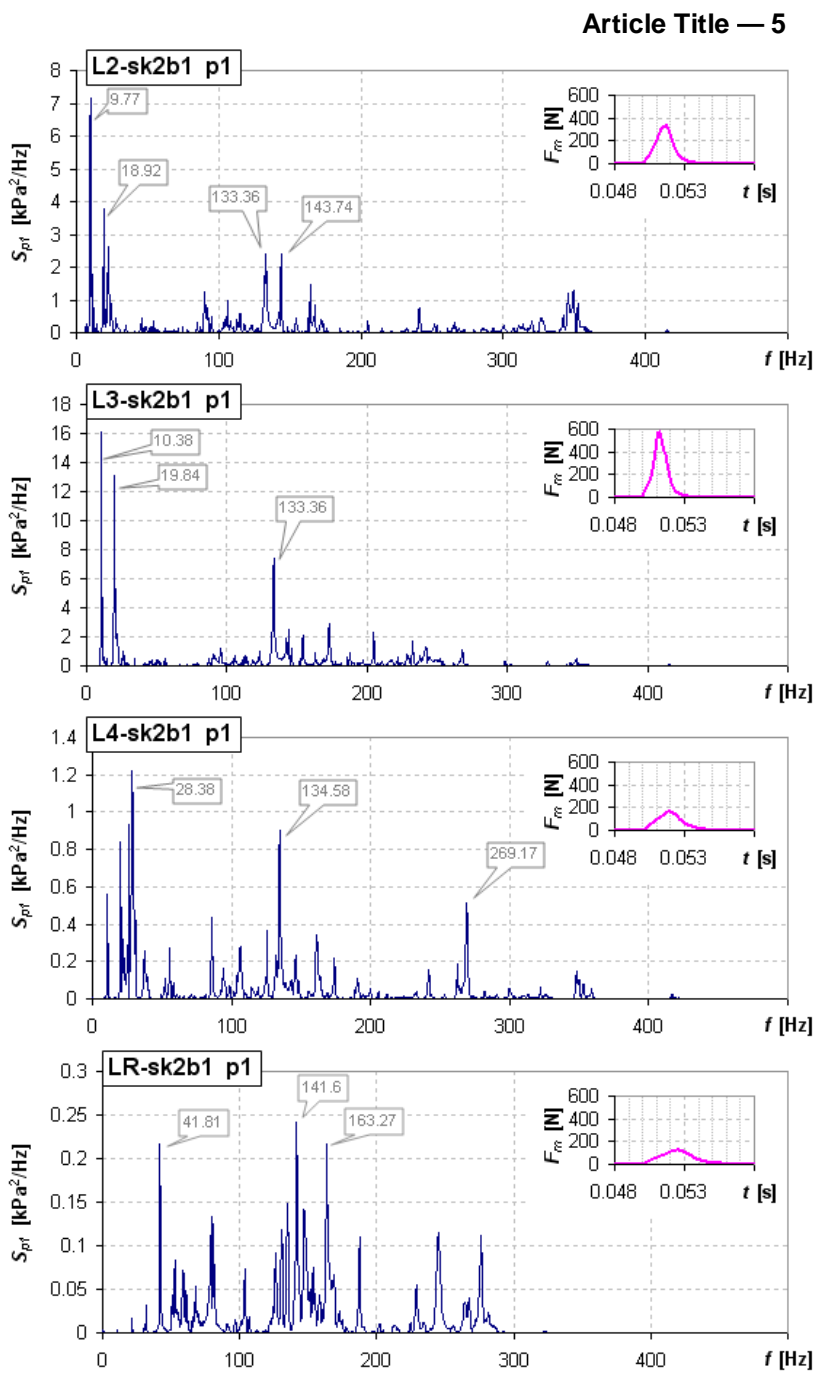

Figure 10. Pressure power spectra of $P 1$ signal produced by longitudinal (1) impact at point B

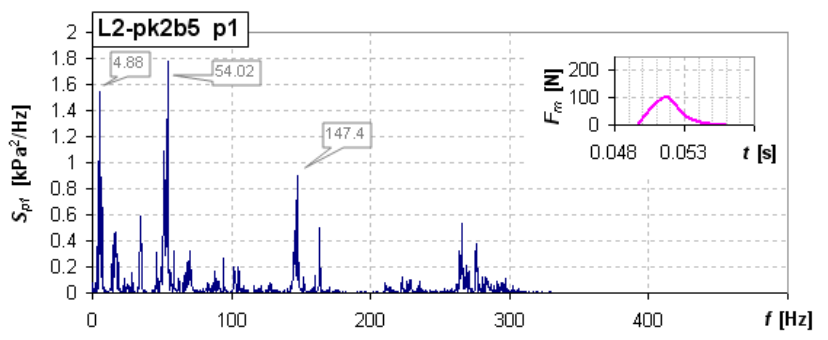

Figure 11. Pressure power spectrum of $P 1$ signal produced by transverse (5) impact at point B

In Figure 12 the power spectra of acceleration signal in transverse direction (DB2) are presented for support system $\mathrm{L} 2$ and various hydraulic $\mathrm{BC}$ - for empty pipe as well as for flowing and standing water. In Figure 13 similar power spectra of acceleration signal in transverse direction (DB2) are presented for supports system L4 and various flow conditions (empty pipe, pipe with standing water, pipe with flowing water). It can be observed that the power spectra for standing and flowing water are similar for both L2 and L4 supporting system and the first frequency peak in power spectrum is lower than for empty pipe. For L2 system the frequencies are $7.02 \mathrm{~Hz}$ and $8.85 \mathrm{~Hz}$ and for $\mathrm{L} 4$ are higher $(7.63 \mathrm{~Hz}$ and $9.77 \mathrm{~Hz})$ due to more rigid supports. 

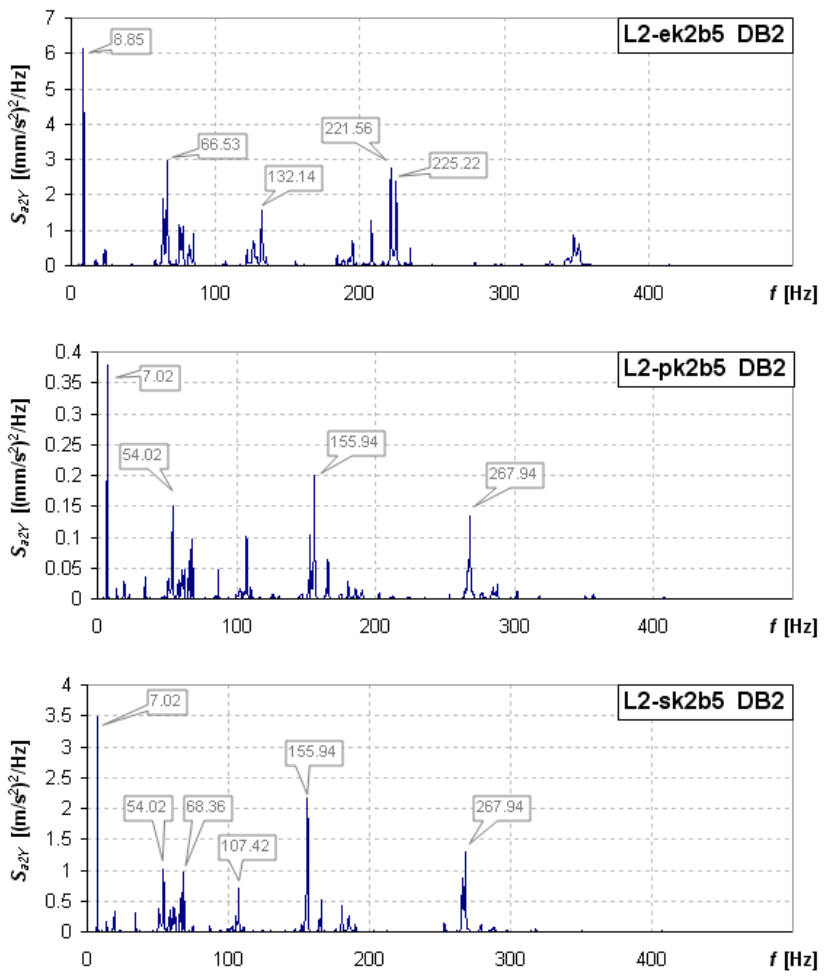

Figure 12. Acceleration power spectrum of DB2 signal produced by transverse (5) impact at point $B$ for $L 2$ supports configuration.
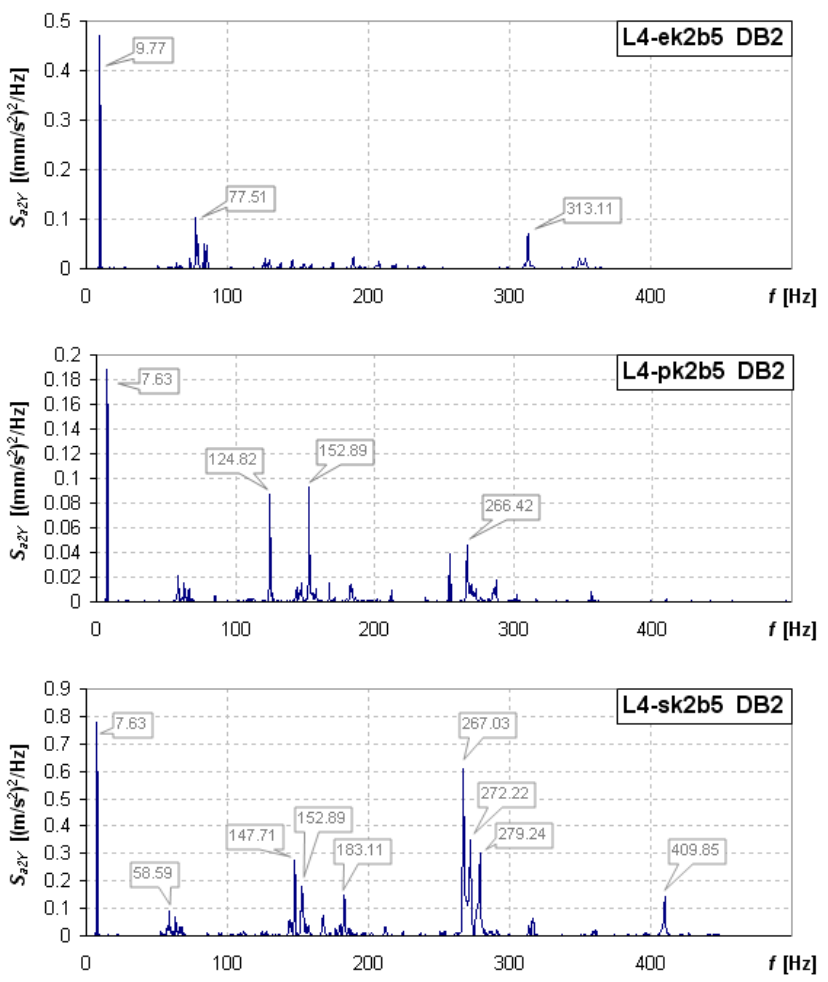

Figure 13. Acceleration power spectrum of DB2 signal produced by transverse (5) impact at point B for $L 4$ supports configuration

Lower frequencies for the pipe with water have validated the assumption used in the standard model of $\mathrm{WH}-\mathrm{FSI}$, that in transverse vibrations water should be accounted as an added mass.

\section{ANALYSES AND DISCUSSION}

Article Title -6

A large amount of data was acquired during experiments and their preliminary analysis was done. The experimental results have allowed to formulate conclusions presented above. In order to achieve the real understanding of the considered phenomenon one has to propose the proper physical interpretation of the results which is consistent with those conclusions. In the current section further discussion, explanation and conclusions are presented.

In order to do additional comparison and interpretation of the logarithmic damping decrements calculated for $\mathrm{WH}$ runs measured for various types of pipeline supporting additional results presented in Figure 14 will be used.

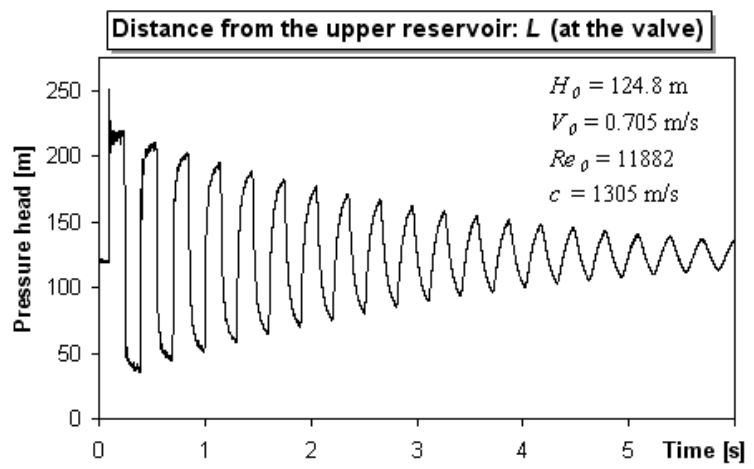

Figure 14. Pressure run at the valve for $98 \mathrm{~m}$ long cooper pipeline of diameter $16 \mathrm{~mm}$ fixed rigidly to the floor

These results are one of the examples of $\mathrm{WH}$ runs investigated for verifying unsteady friction models [9]. The cooper pipeline of the length $L=98 \mathrm{~m}$ and inner diameter $D=16 \mathrm{~mm}$ was fixed quasi rigidly (much more than LR configuration in the present experiment) to the floor. The results for the initial flow velocity $v_{0}=0.705 \mathrm{~m} / \mathrm{s}$ are presented in Figure 14. For these measurements a logarithmic damping decrement can be calculated to be $\delta=0.1035$. In order to compare this value with the previous ones calculated for the diagrams presented in Figure 6 we will assume that the decrement is proportional to the ratio $L / D$. It can be proved that this assumption is valid if pressure losses in one cycle are not large and they are proportional to the product $L / D$ and initial pressure magnitude. Taking into account the current pipeline sizes $(L=59 \mathrm{~m}, D=20 \mathrm{~mm})$ and this assumption the calculated decrement can be rescaled to get the value of $\delta=0.050$. This result is in good consistency with the series of $\delta\left(\delta_{2}=0.096, \delta_{3}=0.091, \delta_{4}=0.083, \delta_{R}=0.069\right)$ found for the runs presented in Figure 6 and the formulated conclusion. One can observe the decrements decrease with increasing the stiffness of the supports and the smallest value ( $\delta=0.050$ after rescaling) is for the most rigid pipe fixing. In fact, the above regularity was found for the detailed stiffness (and configuration) of the supports used at the pipeline and tested during experiments. A wider experimental research could be required to test if this regularity is valid for supports of still lower stiffness. It was also found that for some of the pressure runs lowering of the amplitude was not regular, especially within the initial part of the records. These behaviors are probably the result of coupled pipeline vibrations or other physical effects mentioned before and discussed further. Thus, for effective calculation of the 
damping decrements with Eq.(1) the parameters $k$ and $N$ where usually selected as $k>3$ and $N>10$. The general conclusion of lowering of damping decrements with increasing the support stiffness was confirmed for majority of runs.

The essence is to understand and explain why damping of the pressure magnitudes is smaller when the stiffness of the pipeline supporting system increases. The only reason of such behavior must be larger energy dissipation or spreading for lower stiffness of tested supports. There are two general sources of damping in the current piping system. The first is connected with the structure and the second with the fluid or, in fact, the friction between the liquid and the pipe wall. The former losses can be the result of damping at the supports, other kinds of structural friction or spreading of the energy to the surroundings, especially to the foundation. For the current case the damping at the steel supports is small, however the other two possibilities of structural losses are present and could be significant. Moreover spreading of the energy to the foundation (or supports) should be dependent on the frequency characteristics of the foundation system and the energy transfer is expected to be more effective for better matching of these frequencies to the frequencies of loads $(\mathrm{WH})$. This general regularity was also verified in detailed with numerical investigations [14]. On the other hand the influence of friction between the pipe-wall and the liquid can be significant as well. It is known that the friction is a nonlinear function of the relative liquid velocity $u=v-w$ ( $w$ is the structure velocity and $v$ is absolute liquid velocity), e.g. for quasi-static friction model the losses are proportional to $u^{2}$. For rigid structure $w=0$ and for vibrating pipeline $w \neq 0$, though the time averaged $w$ is zero $(<w>=0)$. Thus the value of $u^{2}$ for the latter case is larger than for rigid structure and the losses are higher for larger vibrations.

A very important characteristics of the wave that should be observed and discussed is the absolute height of the pressure magnitude, especially for the first or a few first pulses. In fact, for the presented runs and tested supports there was no distinct influence of the supports stiffness onto this parameter. Slightly smaller amplitudes for lower stiffness can be observed for the case presented in Figure 7 (P3 pressures). This is probably because the pipe at the very end ( $P 1$ pressures) is fixed rigidly while it is fixed with elastic supports at P3. Numerical investigations done by one of the authors [14] allowed to find out that for the case of pipe with elastic supports there is a possibility of lowering of the (initial) pressure peaks. This could be a kind of shock absorption by the elastic supports. For further oscillations this effect can be reversible and the energy may be transferred back producing additional amplitude increase. This kind of effects can produce irregularities in pressure runs and are possibly to observe at the pressure records within current measurements. However, there is probably another source of such irregularities as well, which can be the complex system of pipeline coupled vibrations.

Beside the height of the amplitude an important parameter of the pressure wave is its shape, which finally reflects the frequency composition of the signal. We can assess the shape directly with a certain method or on the
Article Title -7

basis of the FFT analysis. Such analyses are presented in Figure 8 for the pressure runs displayed in Figure 6. Beside the characteristic frequencies the diagrams show the level of subsequent frequency components which could be an important factor allowing for estimation of the influence of elastic pipe supports. In fact, due to the method of FFT, these diagrams reflects combine influence of absolute initial pressure height and the rate of the amplitude decaying. In general, lowering of the spectrum level for supports of lower stiffness is observed. One can observe higher frequency components of the classic $\mathrm{WH}$ run as well, i.e. odd multiplications of the main WH frequency. Also for these frequencies the power level decreases with decreasing the support stiffness. Moreover, the level of subsequent components drops more sharply for low support stiffness, thus higher components are reduced stronger. The used FFT method of average estimation of pressure variation may have important advantage. If the pressure runs in time are less regular, which has happened for some of the measurements due to the earlier mentioned effects, the average FFT method would allow for more effective analyses of the system behavior. The above analyses also show that transferring of the shape of the wave from rectangular to sinusoidal is a positive effect.

\section{SUMMARY}

In the paper the problem of the influence of elastic pipe supports on the parameters of transient flow is discussed. The basis for the analyses was the measurement results acquired during tests performed at the dedicated experimental stand constructed at the IMP PAN laboratory. Preliminary conclusions were formulated and they are presented throughout the paper. In general it was concluded that for the tested supports lowering of the supports stiffness causes higher reduction of WH pressure oscillations mainly, due to the amplitude damping effect. A few mechanisms of energy dissipation were proposed to explain this behavior. They were liquid pipe-wall friction and $\mathrm{WH}$ energy transfer and dissipation at the structure. In fact, due to additional analyses made it seems that, lowering of the stiffness could be considered to be effective because of producing larger pipeline vibrations and better energy spreading and dissipation at the structure. It can be also expected, that supports of higher damping properties could be more effective in reduction of pressure amplitudes, due to larger energy dissipation. It was also found that lower stiffness of the supports caused mitigation and faster disappearing of the column separation effects.

The classical WH theory cannot predict the above mentioned effects. Therefore for numerical modeling it is necessary to use FSI based models. In general the considered problem is complex and requires further analyses and investigations especially within the effects mentioned above. Currently the role of damping at the pipe supports is expected to be of special interest. A more detailed examinations of $\mathrm{WH}$ with $\mathrm{CS}$ is intended as well. Further numerical modeling and comparison of the computational results with experiments are also planned. 


\section{ACKNOWLEDGMENTS}

The experimental results presented in the paper were acquired within the research project number $\mathrm{N} N 504$ 478839, funded by the Ministry of Science and Higher Education of Poland.

\section{REFERENCES}

[1] E.B. Wylie, V.L. Streeter. Fluid transients in systems, NJ, Prentice-Hall, 1993.

[2] A.S. Tijsseling, C.S.W. Lavooij. Waterhammer with fluid-structure interaction, Applied Scientific Research, 47, pp. 273-285, 1990.

[3] D. Wiggert, A. Tijsseling. Fluid transients and fluidstructure interaction in flexible liquid-filled piping. Applied Mechanics Review, 54, 9: 455-481, 2001.

[4] S. Henclik. A numerical approach of the standard model of water hammer with fluid-structure interaction. Journal of Theoretical and Applied Mechanics. 53, 3, pp. 543-555. DOI: 10.15632/jtampl.53.3.543, 2015.

[5] D. Wiggert, R.S. Otwell, F.J. Hatfield. The effect of elbow restraint on pressure transients. Trans. of the ASME, 107, 9, pp. 402-406, 1985.

[6] A.G. Heinsbroek, A.S. Tijsseling. The influence of support rigidity on water hammer pressures and pipe stresses, Proc. 2nd Int. Conf. Water in Pipe. Syst. Edinburgh, 1994.

[7] A. Adamkowski, S. Henclik, M. Lewandowski. Experimental and numerical results of the influence of dynamic Poisson effect on transient pipe flow parameters. 25 IAHR Symposium on Hydraulic Machinery and Systems, Timisoara. IOP Conf. Series: Earth and Environmental Sciences. DOI: 10.1088/1755-1315/12/1/012041, 2010.

[8] A. Bergant, A.R. Simpson, J. Vitkovsky. Developments in unsteady pipe flow friction modeling. J. Hydraulic Research, 39, 3, pp. 249-257, 2001.

[9] A. Adamkowski, M. Lewandowski. Experimental examination of unsteady friction models for transient pipe flow simulation. J. Fluids Eng., ASME 128 (6), 1351-1363, 2006.

[10] D.D. Budny, F. J. Hatfield, D.C. Wiggert. An experimental study on the influence of structural damping on internal fluid pressure during a transient flow. ASME J. Pressure Vessel Technology, 112, 8, pp. 284-290, 1990.

[11] A. Keramat, A.S. Tijsseling, Q. Hou, A. Ahmadi. Fluidstructure interaction with pipe-wall viscoelasticity during water hammer. J. Fluids Struct. 28, pp. 434-455, 2012.

[12] A. Adamkowski, M. Lewandowski. The Pressure Surge Damping Decrement and the Pressure Traces Convergence Rate as the Parameters Applied to Evaluate the Friction Models Used in WaterHammer Prediction. Proc. 33rd Int. Association of Hydraulic Engineering and Research Congress. 6019-6026. Curran Associates. Red Hook, NY 2009.

[13] A. Adamkowski, M. Lewandowski. Investigation of Hydraulic Transients in a Pipeline with Column Separation. J. Hydraulic Eng. 138(11), pp. 935-944,
2012.

[14] S. Henclik. Numerical study on water hammer with fluid-structure interaction in a straight pipeline fixed with viscoelastic supports. Proc. $3^{\text {rd }}$ Polish Congress of Mechanics and $21^{\text {st }}$ International Conference on Computer Methods in Mechanics. Gdansk 2015. 